\title{
POWER GRID SYNCHRONIZATION FAILURE DETECTION AND RECOVERY
}

\author{
Pankaj Singh ${ }^{1}$, Anuj Sharma ${ }^{2}$, Shubham Saha ${ }^{3}$, Anshul Chauhan ${ }^{4}$, Amit Verma ${ }^{5}$ \\ 1,2,3,4,5 Department of Electronics and Communication Engineering, S.R.M University, NCR Campus, Ghaziabad, U.P
}

\begin{abstract}
As the power grid failure problems continue to persist in the country, need arises to make it more efficient and effective. This is a demonstration devised to provide such kind of a system that could detect the failure in synchronous working of the power grid in case any external supply source that is supplying to the grid is encountering any kind of abnormalities may be in current levels, voltage levels, temperature and oil level. There are various generation units that usually supply power to the grids such as thermal plants, solar plants, hydro-electric plants etc. All the generation units that connect to the grid have to obey the rules or standards laid down for the operation of a grid. These rules involve maintaining of voltage variation, current variation, oil-level variation and temperature variation within these specified limits. If there are any kind of abnormalities or deviations from the acceptable limits laid down by the grid, it then gets crucial that the particular feeding source should get automatically disconnected from the entire grid temporarily, this process is widely termed islanding. This would prevent large scale occurrences of events of black outs. Hence it would be beneficial and wise if we could have a system that would be so efficient that it could issue intimations about the potential variations in the arrangements and in addition provide the exact distance of the fault location on a feeder line from the base grid and keep the particular source temporarily on a standby basis while this process takes place. Variation of the system voltage should be around $+-5 \%$ as per central electricity authority of India Regulations, 2010. This paper is an approach on devising out a system that helps the grid resolve problems related to black out situations hence making it a smart grid.
\end{abstract}

Keywords: - current variation, voltage variation, temperature variation, oil level detection, islanding, smart grid, active method, passive method.

\section{INTRODUCTION}

An approach towards a smarter tomorrow is always an achievement and this paper discusses how this approach helps make our power grid's functioning more enhanced and reliable. The problem of islanding is a common problem when we talk about the distribution system and hence methods that have been devised for the prevention of possible islanding situation are grouped into following categories:

- Passive methods.

- Active methods.

These methods and their performance is characterized by certain requirements that have been laid down by the Institute of Electrical and Electronics Engineering (IEEE), International Electro Technical Commission (IEC) and many other Standards Organizations. As far as active methods are concerned, minor disturbances are introduced into the power system and thereafter the response that the system provides is observed and monitored. These methods result in alteration of the balance of power existing amid generators and the load, hence reducing the quality of the power that we obtain from the system. There are several examples of the active method a few of them being impedance measuring method, AFD-Active frequency drift, AFDPF-Active frequency drift with positive feedback, APS-
Automatic phase shift and ALPS-Adaptive logic phase shift. Whereas on the other hand passive methods continuously monitors various parameters of the power grid like in this case over voltage, over current, temperature variation and oil level indication.

\section{OPERATIONAL PRINCIPLE}

In passive method the parameters may vary differently affecting the working of the grid. Hence any source that violates the rules of grid would be immediately terminated. But the process will not stop there because just elimination is not enough, we need to know the reason of the fault i.e which parameter is showing variation is it current, voltage or any other and also we need to determine what the location of the fault site is from the base of grid. Since our demonstration here depicts 3 different sources as shown in Fig.1, meanwhile if one source misbehaves the load will be shared by the other two sources that are connected to the grid hence the transmission will be uninterrupted. Hence the concept of load sharing is quite a crucial part of this demonstration. At the same time the entire faulty line is being examined for detection of the cause of the error as well as the distance of that particular fault location. 


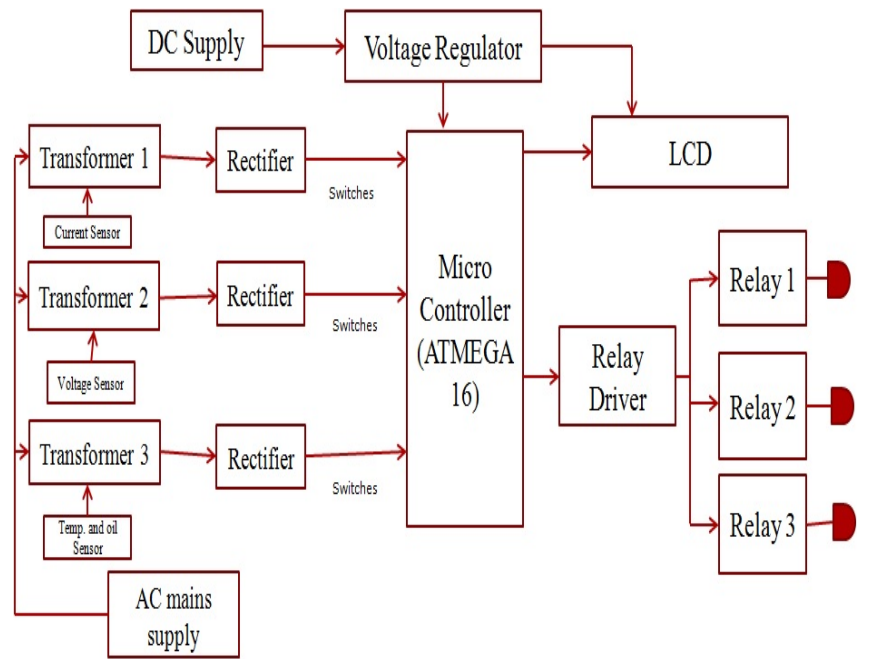

Fig.1 Basic block depicting sources, line and grid base.

The microcontroller section acts as the power grid hub. This section will continuously monitor the entire system. As shown in Fig. 1 different sensors have been used. The current sensor measure the variation in current values in case a shorted or an open line and after that it forwards this information to the controller, which intern determines with a predetermined threshold level weather to cut off the source or not. The voltage sensor also operates in the similar fashion. Temperature is another parameter which is responsible for grid failures in case it goes out of desired ranges, hence here the sensor would monitor the temperature of the transformer and any variation in current levels will trigger a change in the temperature which the sensor would detect and convey the information to controller, which again will compare it to a predetermined threshold value of temperature and decide if to terminate the line or not. The oil level indication also works on the same principle as above and determines if the oil level has reduced drastically below the unaccepted range and alerts the grid about the same.

After the cause of fault has been determined and displayed at the grid hub one more parameter is there that can be determined and can add up I easing the complexity of recovery and maintenance and that is the distance calculation of a shorted or an open point on the feeder line coming from three different sources. The fault can be anywhere in the line hence being able to determine the exact distance of the point where the fault has occurred is a task that would add up to the effective management of the grid. For this purpose switches can be used at, say each kilometer and the on and off state of a switch could help determine the fault location. Hence calculated distance can be provided at the grid base station and physical maintenance can be initiated within no time.

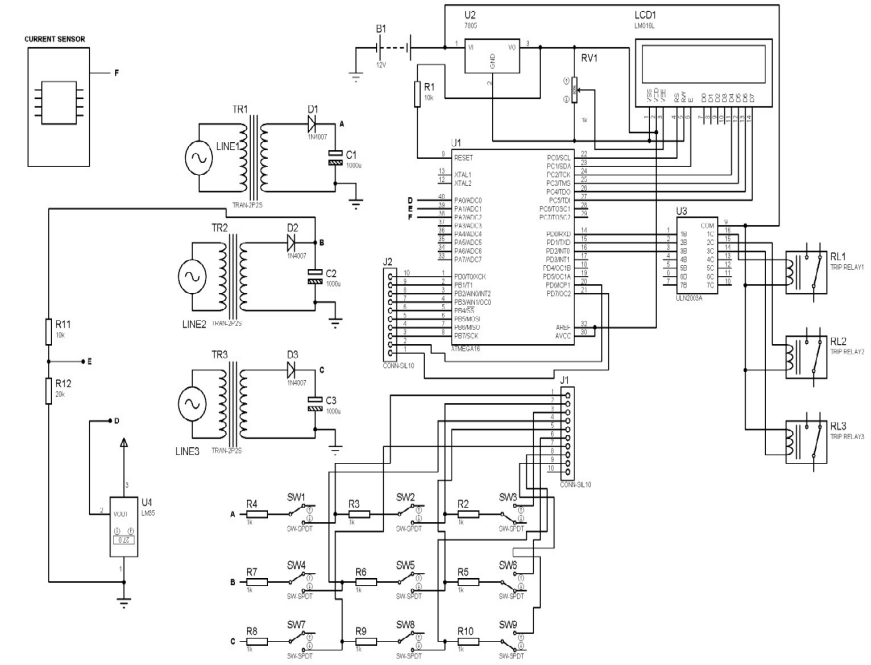

Fig.2 Circuit diagram of the actual implementation

Fig.2 given above is a generalized circuit diagram for the implementation of above mentioned concept. Fig.2 comprises of the entire section starting from the transformer section that shows three different sources supplying power, and then comes the transmission lines section that consists of three different lines connecting to the controller separately, these lines are being continuously monitored by the sensors namely a current sensor, voltage sensing unit, oil level detection and temperature sensing unit which have been depicted in the leftmost section of fig.2. The controller section however acts as the grid base, taking all the crucial decisions regarding the safety of the grid.

Whenever the controller section encounters any variation in the predetermined limits set for each of the parameters it issues a signal that is directed to the LCD section and that particular alert message is then displayed and at the same time the source that has been creating this abnormality is also detected and temporarily eliminated from the grid hence safeguarding the failure of entire grid. The relay unit

has been employed for the purpose of elimination of the disturbing source temporarily. The switches on the other hand that have been employed on the transmission line section are the fault detection switches that would let us calculate the distance to the fault site, the controller section would be providing this information to the display unit hence telling us the exact distance of the occurrence of a shorted line or an open line accordingly hence the maintenance work can be started thereafter.

\section{EFFECTIVE MANAGEMENT RECOVERY}

Maintaining a power grid online $24 \mathrm{hrs}$ and keeping it efficient and effective at the same time is quite a tough task seeing the complexity of the grids. Hence our implementation offers a way in which it would become quite effective to run the maintenance process and recover the grid back into normal functioning in cases of occurrences of faults. 
This implementation would yield following outcomes:

- Current overload detection.

- Voltage overload detection.

- Temperature variation \& oil level indication.

- Distance determination from grid to fault site.

With all this precise information at the doorsteps of the grid base it becomes convenient to run the maintenance and recovery tasks. Knowing the fault location precisely enables the physical workforce to manually correct it and at the same time when the fault in line is corrected the grid would immediately sense this and the source and the feeder line that was previously terminated would be again recovered and connected back to the grid hence restoring the entire balance.

\section{REALISTIC CONSTRAINTS}

- When we talk about distribution lines, one major drawback is they are earthed at the substations, so when the generation unit and lines get disconnected the line no longer remains earthed hence causing voltage overloads.

- If the distribution lines are short circuited, then the large amount of current will flow leading to current overload.

- Due to islanding one major problem grid faces is that of establishing a reconnection with the source as at that time both source and grid might be out of synchronization hence resulting in huge power flows that could possibly cause damage to grid, to lines, stations and consumer units.

\section{CONCLUSION}

This implementation concludes that it is possible to have a power grid system that is smarter, more effective as well as efficient in its operation, thus proving to be more economical as compared to be the present installations. The challenge is a continuous and uninterrupted transmission which can be very well achieved with the implementation described by this paper and in addition to the continuous transmission several other parameters i.e. the passive parameters are being monitored regularly and any discrepancies occurring in these, are taken into account and accordingly worked upon thus making the process of management and recovery convenient and effective.

\section{ACKNOWLEDGEMENT}

A sincere gratitude towards our mentor and guide $\mathrm{Mr}$. Pankaj Singh who has been always a great motivator and a great help and we would like to acknowledge our project cocoordinators for their valuable contributions in drafting and accomplishment of this proposal. Also we would like to express our utter thanks to them for sharing their wisdom with us.

\section{REFERENCES}

[1]. J.Shortle, Steffen Rebennack, Fred, W.Glover, Syst. Eng. \& Oper. Res., George Mason Univ., Fairfax, VA, United States. IEEE paper on "Transmission-Capacity Expansion for Minimizing Blackout Probabilities", 13 September 2013.

[2]. Sabita Maharaj, Quanyan, Yan Zang, Stien Gjessing, Tamer Basar, Simula Res. Lab., Formebu, Norway. IEEE paper on "Response management in smart grids in largely populated regions" IEEE Transactions on Smart Grid (vol:7, Issue: 1), 1 June 2015

[3]. Moussa Reda Mansour, Luis Fernando, Costa Alberto, Rodrigo Andrade Ramos, University of Sao Carlos, Brazil. IEEE paper on "Preventive control design for voltage stability considering multiple contingencies". IEEE Transcriptions on Power Systems (vol: 31, Issue: 2), 30 April 2015.

[4]. Shuhui Li, Julio Proano, Dong Zang. IEEE paper on "Micro grid power flow study in grid connected and islanding modes under converter control strategies". IEEE 2012. 